Elliptic flow of thermal photons in heavy-ion collisions at energies available at the BNL Relativistic Heavy Ion Collider and at the CERN Large Hadron Collider

Holopainen, $\mathrm{H}$.

2011

Holopainen, H , Räsänen , S S \& Eskola , K J 2011 , ' Elliptic flow of thermal photons in heavy-ion collisions at energies available at the BNL Relativistic Heavy lon Collider and at the CERN Large Hadron Collider' ', Physical Review C (Nuclear Physics) , vol. 84 , no. 6 , pp. 064903 . https://doi.org/10.1103/PhysRevC.84.064903

http://hdl.handle.net/10138/32700

https://doi.org/10.1103/PhysRevC.84.064903

publishedVersion

Downloaded from Helda, University of Helsinki institutional repository.

This is an electronic reprint of the original article.

This reprint may differ from the original in pagination and typographic detail.

Please cite the original version. 


\title{
Elliptic flow of thermal photons in heavy-ion collisions at energies available at the BNL Relativistic Heavy Ion Collider and at the CERN Large Hadron Collider
}

\author{
H. Holopainen, ${ }^{*}$ S. S. Räsänen, ${ }^{\dagger}$ and K. J. Eskola ${ }^{\ddagger}$ \\ Department of Physics, P.O. Box 35, FIN-40014 University of Jyväskylä, Finland and \\ Helsinki Institute of Physics, P.O. Box 64, FIN-00014 University of Helsinki, Finland \\ (Received 1 May 2011; revised manuscript received 12 September 2011; published 2 December 2011)
}

\begin{abstract}
We calculate the thermal photon transverse momentum spectra and elliptic flow in $\sqrt{s_{N N}}=200 \mathrm{GeV} \mathrm{Au}+\mathrm{Au}$ collisions at the Relativistic Heavy Ion Collider (RHIC) and in $\sqrt{s_{N N}}=2.76 \mathrm{TeV} \mathrm{Pb}+\mathrm{Pb}$ collisions at the Large Hadron Collider (LHC), using an ideal hydrodynamical framework which is constrained by the measured hadron spectra at RHIC and LHC. The sensitivity of the results to the QCD-matter equation of state and to the photon emission rates is studied, and the photon $v_{2}$ is discussed in light of the photonic $p_{T}$ spectrum measured by the PHENIX Collaboration. In particular, we make a prediction for the thermal photon $p_{T}$ spectra and elliptic flow for the current $\mathrm{LHC} \mathrm{Pb}+\mathrm{Pb}$ collisions.
\end{abstract}

DOI: 10.1103/PhysRevC.84.064903

PACS number(s): 12.38.Mh, 25.75.Cj, 25.75.Ld

\section{INTRODUCTION}

Experimental data at the Relativistic Heavy Ion Collider (RHIC) and now also at the Large Hadron Collider (LHC) have shown compelling evidence of strongly interacting QCDmedium production in ultrarelativistic heavy-ion collisions. The measured transverse energies, transverse momentum $\left(p_{T}\right)$ spectra, and, in particular, the significant azimuthal anisotropy (elliptic flow) of final-state hadrons suggest together that partonic QCD matter, quark-gluon plasma (QGP), is formed in these collisions.

In the collision of two nuclei, the azimuthally anisotropic overlap region sets preferred directions for the transverse flow. In hydrodynamical models, pressure gradients turn the spatial anisotropy of the produced hot matter into a flow anisotropy, which is transmitted into the momentum distributions of measurable final-state hadrons at the decoupling of the system. However, the hadronic measurement reflects the flow (and temperature) conditions only in the freeze-out region where the hadronic interactions cease.

In comparison with partons, photons interact only very weakly with the QCD matter and thus a photon emitted from the medium most likely escapes from the system without interacting. This is seen also in the measurements where photons do not show a similar suppression as hadrons when we move from proton+proton $(p+p)$ to nucleus+nucleus $(A+A)$ collisions [1]. Since photons can escape from the medium without interacting, they carry information about the system at the time of their production.

Ultrarelativistic heavy-ion collisions are particularly interesting in regard to direct photon production, since relative to $p+p$ collisions there are different types of nuclear effects at work as well as a number of important further sources for photons. In $p+p$ (and also in $p+A$ ) collisions the direct photons are prompt photons originating from the primary hard

\footnotetext{
*hannu.1.holopainen@jyu.fi

†sami.s.rasanen@jyu.fi

${ }^{\ddagger}$ kari.eskola@phys.jyu.fi
}

interactions of partons, and fragmentation photons emitted by the primarily produced high- $p_{T}$ partons [2]. In heavy-ion collisions (and also in $p+A$ collisions), both the prompt and fragmentation photons at high $p_{T}$ are subjected to nuclear effects in the parton distribution functions of the colliding nuclei (see, e.g., Refs. [3,4] for the quantification of these effects and their uncertainties). The fragmentation photon component, however, is expected to be suppressed due to the quenching of partonic jets in QCD matter in $A+A$ collisions. In addition to this, in $A+A$ collisions the jet (parton)matter interactions, i.e., the jet-photon conversion $[5,6]$ and collision-induced photon emission from high-energy partons, can produce photons which are important in the mid- $p_{T}$ region [7-9]. Finally, the hot medium itself emits thermal photons, which are expected to be important in the few-GeV region and below, as discussed in the hydrodynamical studies of Refs. [7,8,10-13].

In heavy-ion collisions, it is very difficult to distinguish between the different direct photon sources. In addition, there is a huge decay-photon background to deal with. The elliptic flow of direct photons could, however, shed more light on the interplay of the various photon production sources, which differ from each other as follows: At high $p_{T}$ (above $\sim 5 \mathrm{GeV}$ at RHIC), where prompt photons dominate [7] and where the fragmentation photons are more suppressed in the outof-plane direction (perpendicular to the impact parameter), the photonic $v_{2}$ should be positive but very small [9]. The jet-medium interactions in turn increase the photon production most strongly in the in-plane direction (parallel to the impact parameter), thus causing a negative $v_{2}$ contribution at mid $p_{T}$ [9]. The thermal photon production is affected by the hydrodynamical transverse flow itself, so photons in the in-plane direction get a stronger boost. As shown earlier in Refs. [14-17], this results in a positive elliptic flow for the thermal photons. Because the net contribution from other sources to photon $v_{2}$ is expected to be very small or even negative [9], a large (hadron-like) photon $v_{2}$ measured in the few- $p_{T}$ region and below should thus serve as a signature of thermal photon dominance. Since QCD matter is emitting photons throughout its entire evolution, measuring thermal 
photon $p_{T}$ spectra and $v_{2}$ would thus give important further constraints for the dynamics and properties of QCD matter.

In this work we focus on computing the thermal photon $p_{T}$ spectra and elliptic flow in $\sqrt{s_{N N}}=200 \mathrm{GeV} \mathrm{Au}+\mathrm{Au}$ collisions at RHIC and in $\sqrt{s_{N N}}=2.76 \mathrm{TeV} \mathrm{Pb}+\mathrm{Pb}$ collisions at the LHC, using an ideal hydrodynamical framework which is constrained by the measured hadron spectra at RHIC and LHC. We study the sensitivity of the results to the QCD-matter equation of state (EoS) and to the photon emission rates. We discuss the photon $v_{2}$ in light of the photonic $p_{T}$ spectrum measured by the PHENIX Collaboration [18-20]. In particular, we make a prediction for the thermal photon $p_{T}$ spectra and elliptic flow for the current LHC heavy-ion collisions. Previous predictions for the thermal photon $p_{T}$ spectra in $\mathrm{Pb}+\mathrm{Pb}$ collisions at the planned maximum center-of-mass energy of 5.5 TeV of the LHC can be found in Refs. [21,22].

\section{THEORETICAL FRAMEWORK}

\section{A. Centrality classes}

Centrality classes for $A+A$ collisions studied here are calculated using the optical Glauber model. For nuclear densities we use spherically symmetric Woods-Saxon profiles with the the thickness parameter $d=0.54 \mathrm{fm}$ and radii $R_{\mathrm{Au}}=6.37 \mathrm{fm}$ and $R_{\mathrm{Pb}}=6.49 \mathrm{fm}$. The total cross section for $A+A$ collisions is calculated from

$$
\sigma_{\mathrm{tot}}^{A A}=\int d^{2} \boldsymbol{b} \frac{d \sigma_{\mathrm{tot}}}{d^{2} \boldsymbol{b}}=\int d^{2} \boldsymbol{b}\left(1-e^{-T_{A A}(\boldsymbol{b}) \sigma_{N N}^{\mathrm{in}}}\right),
$$

where $T_{A A}$ is the standard nuclear overlap function and $\sigma_{N N}^{\text {in }}$ is the inelastic nucleon-nucleon cross section. We take $\sigma_{N N}^{\text {in }}=$ $42(64) \mathrm{mb}$ for $\sqrt{s_{N N}}=200$ (2760) GeV.

The centrality classes are defined with impact parameter ranges $\left[b_{i}, b_{i+1}\right]$ so that for the centrality class of $c_{i}$ we have

$$
c_{i}=\frac{1}{\sigma_{\mathrm{tot}}^{A A}} \int_{b_{i}}^{b_{i+1}} d^{2} \boldsymbol{b}\left(1-e^{-T_{A A}(\boldsymbol{b}) \sigma_{N N}^{\mathrm{in}}}\right) .
$$

The average impact parameter for each centrality class is calculated using the distribution $d \sigma / d^{2} b$ as a weight. The average number of participants is calculated similarly. The obtained centrality classes, impact parameter ranges, average impact parameters, and number of participants are listed in Table I.

\section{B. Initial states for hydrodynamical evolution}

For RHIC we use the EKRT saturation model [23] to fix the initial entropy in most central collisions. As shown in Refs. [24,25] we can get a good description of the pion spectra and the elliptic flow with this pQCD + saturation + hydrodynamics approach. For $\mathrm{Au}+\mathrm{Au}$ collisions at $\sqrt{s_{N N}}=$ $200 \mathrm{GeV}$ the model gives an initial time $\tau_{0}=0.17 \mathrm{fm}$. For the $\sqrt{s_{N N}}=2.76 \mathrm{TeV} \mathrm{Pb}+\mathrm{Pb}$ collisions at the $\mathrm{LHC}$, we fix the initial entropy so that we reproduce the measured multiplicity [26]. The initial time $\tau_{0}=0.12 \mathrm{fm}$ is based on the EKRT-motivated fit performed in Ref. [27].
TABLE I. Various centrality classes for $\mathrm{Au}+\mathrm{Au}$ collisions at $\sqrt{s_{N N}}=200 \mathrm{GeV}$ and for $\mathrm{Pb}+\mathrm{Pb}$ collisions at $\sqrt{s_{N N}}=2.76 \mathrm{TeV}$, obtained via the optical Glauber model.

\begin{tabular}{ccccc}
\hline \hline & $\begin{array}{c}\text { Centrality } \\
(\%)\end{array}$ & $\begin{array}{c}\text { Range of } b \\
(\mathrm{fm})\end{array}$ & $\begin{array}{c}\langle b\rangle \\
(\mathrm{fm})\end{array}$ & $N_{\text {part }}$ \\
\hline RHIC & $0-5$ & $0.00-3.35$ & 2.24 & 346 \\
& $5-10$ & $3.35-4.74$ & 4.08 & 289 \\
& $10-15$ & $4.74-5.81$ & 5.30 & 242 \\
& $15-20$ & $5.81-6.71$ & 6.27 & 202 \\
& $20-30$ & $6.71-8.21$ & 7.49 & 153 \\
& $30-40$ & $8.21-9.49$ & 8.87 & 102 \\
& $40-50$ & $9.49-10.6$ & 10.1 & 64.4 \\
& $50-60$ & $10.6-11.6$ & 11.1 & 37.5 \\
& $0-20$ & $0.00-6.71$ & 4.47 & 267 \\
LHC & $20-40$ & $6.71-9.49$ & 8.18 & 128 \\
& $0-5$ & $0.00-3.53$ & 2.35 & 375 \\
& $0-20$ & $0.00-7.05$ & 4.70 & 294 \\
& $20-40$ & $7.05-9.98$ & 8.60 & 141 \\
\hline \hline
\end{tabular}

To fix the initial transverse density profile in $\sqrt{s_{N N}}=$ $200 \mathrm{GeV} \mathrm{Au}+\mathrm{Au}$ collisions, we do the following: In Fig. 1 we show, from Ref. [28], the measured charged-particle multiplicity (divided by the number of participant pairs) as a function of the number of participants calculated from the optical Glauber model. ${ }^{1}$ Choosing the initial transverse density according to the binary-collision-scaled energy or entropy density (eBC, sBC), or wounded-nucleon-scaled energy or entropy density (eWN, sWN) as introduced in Ref. [29], we compute the charged-particle multiplicity in the centrality classes obtained earlier. The initial entropy at $b=0$ in these four cases is kept fixed. We see that the sWN profile fits the measured centrality dependence quite well. We therefore choose the sWN profile at RHIC and, for simplicity, use the same profile also for the $\mathrm{LHC} \mathrm{Pb}+\mathrm{Pb}$ collisions.

\section{Hydrodynamics and freeze-out}

To describe the space-time evolution of the produced QCD matter, we solve the ideal hydrodynamical equations

$$
\partial_{\mu} T^{\mu \nu}=0,
$$

where $T^{\mu \nu}=(\epsilon+P) u^{\mu} u^{\nu}-P g^{\mu \nu}$ is the energy-momentum tensor, $u^{\mu}$ is the fluid four-velocity, $\epsilon$ is the energy density, and $P$ is the pressure. Because we are interested in particle and photon production at midrapidity, we may assume that net-baryon density is negligible. Because the particle spectra are approximately flat at midrapidity, we can simplify our hydrodynamical equations by assuming longitudinal boost invariance. We use the SHASTA algorithm [30,31] to solve this $(2+1)$-dimensional numerical problem.

To close the hydrodynamic equations we need an equation of state, $P=P(\epsilon)$. In this paper we study the sensitivity of thermal photon production to the EoS by focusing on two

\footnotetext{
${ }^{1}$ Note that the number of participants quoted by the experiments here is usually from the Monte Carlo Glauber model.
} 


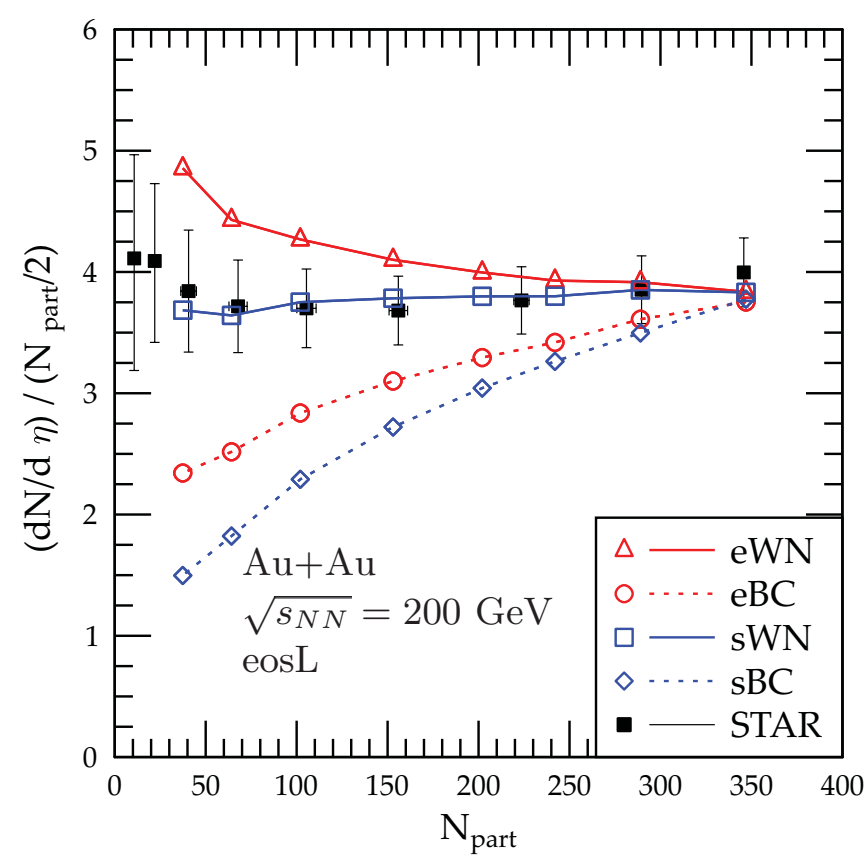

FIG. 1. (Color online) Number of charged hadrons at midrapidity in $\sqrt{s_{N N}}=200 \mathrm{GeV} \mathrm{Au}+\mathrm{Au}$ collisions scaled by the number of the participant pairs calculated from the optical Glauber model. The data are from the STAR Collaboration [28].

different cases. The first case, called here "eosQ," corresponds to the bag-model EoS with a first-order phase transition [32]. In eosQ, the high-temperature phase with the bag constant is an ideal gas of three flavors of massless quarks and gluons, while the low-temperature phase is an ideal gas of all hadronic states with $m<2 \mathrm{GeV}$. These two phases are connected with a mixed phase, and the bag constant is chosen so that the critical temperature is $T_{c}=165 \mathrm{MeV}$. The second EoS case, which we call "eosL," is adopted from Ref. [33]. This EoS is quite similar to the recently constructed lattice EoS "s95-p" [34], and, as discussed in Ref. [34], the hadron spectra and elliptic flow are in practice insensitive to the differences between eosL and s95-p.

Since the lattice data suggest that the phase transition from the QGP to hadron gas (HG) is not of first order, one may consider the eosQ case somewhat unrealistic. However, the computation of thermal photon production in the phasetransition region requires well-defined QGP and hadron-gas fractions, which are available only in the eosQ case. With eosL, in the absence of such phase fractions, there are additional uncertainties in the thermal photon calculation related to the QGP and HG emission rates.

Thermal transverse momentum $\left(p_{T}\right)$ spectra of hadrons are obtained using the Cooper-Frye method [35], where particle emission from a freeze-out hypersurface $\sigma$ is calculated with

$$
\frac{d N^{f}}{d^{2} p_{T} d y}=\int_{\sigma} f(x, p) p^{\mu} d \sigma_{\mu},
$$

where $f(x, p)$ is the momentum distribution function of a specific hadron type. We assume the system to decouple at a single constant temperature $T_{\mathrm{dec}}$, which is fixed so that we get a good agreement with the measured $p_{T}$ spectra of pions at
RHIC. With eosQ, we have $T_{\mathrm{dec}}=140 \mathrm{MeV}$, and $160 \mathrm{MeV}$ with eosL.

After the thermal emission of particles from the freezeout surface is calculated, we take into account the strong and electromagnetic two- and three-body decays. This treatment is essential because most of the stable particles in our case come from the decays of heavy resonances.

The $p_{T}$ spectra of hadrons can be written as a Fourier series,

$$
\frac{d N}{d^{2} p_{T} d y}=\frac{1}{\pi} \frac{d N}{d p_{T}^{2} d y}\left(1+\sum_{n=1}^{\infty} 2 v_{n} \cos (n \phi)\right)
$$

where $\phi$ is the hadron momentum's azimuthal angle with respect to the reaction plane defined by the impact parameter. Elliptic flow, $v_{2}$, is the second coefficient in this series and it can be computed from

$$
v_{2}\left(p_{T}\right)=\frac{\int d \phi \cos (2 \phi) \frac{d N(b)}{d p_{T}^{2} d \phi d y}}{\int d \phi \frac{d N(b)}{d p_{T}^{2} d \phi d y}} .
$$

Correspondingly, the $p_{T}$-integrated $v_{2}$ becomes

$$
v_{2}=\frac{\int d \phi \cos (2 \phi) \frac{d N(b)}{d \phi d y}}{\int d \phi \frac{d N(b)}{d \phi d y}} .
$$

\section{Thermal photons emission from the hydrodynamical medium}

The $p_{T}$ spectra of thermal photons can be calculated from

$$
\frac{d N^{\gamma}}{d^{2} p_{T} d y}=\int d^{4} x \Gamma\left(E^{*}(x), T(x)\right),
$$

where $\Gamma\left(E^{*}, T\right)$ is the Lorentz invariant thermal photon emission rate, $d^{4} x$ is the volume element, and $E^{*}(x)=p^{\mu} u_{\mu}(x)$ is the photon energy in the fluid's local rest frame. For the QGP, we use the emission rate from Refs. [36,37] with $N_{f}=3$ and a running strong coupling constant [38] $\alpha_{s}=\beta / \ln (8 T / \Lambda)$, with $\beta=6 \pi /\left(33-2 N_{f}\right)$ and $\Lambda=200 \mathrm{MeV}$. For the hadron gas, we use two different emission rates: (i) The first are those calculated in Refs. [39,40] and parametrized in Refs. [40,41], which we call "R92." Also the $a_{1}$ exchange in $\pi+\rho \rightarrow \rho+\gamma$ is taken into account as suggested in Ref. [42]. These rates were used in the previously published LHC predictions [21]. (ii) The second are the more recent ones from Ref. [43] which account also for the finite size of hadrons through form factors. We call these rates "TRG."

With eosL, which smoothly goes from the QGP to the HG phase without specifying their volume fractions, one needs to choose how to switch from the QGP to HG photon emission rates. For simplicity, we choose to do this at a constant temperature $T_{s}$ but we vary $T_{s}$ between 170 and $200 \mathrm{MeV}$. We label these two cases as "eosL170" and "eosL200." We emphasize that instead of an ad hoc modeling of volume fractions, we consider these two limiting cases to get a handle on the uncertainties related to the rate-switching procedure.

To illustrate the differences between the R92 and TRG emission rates, which are important for the photonic $v_{2}$ results presented in Sec. III, we plot in Fig. 2 the ratio of the photon emission rates in the HG and QGP at two different fixed 


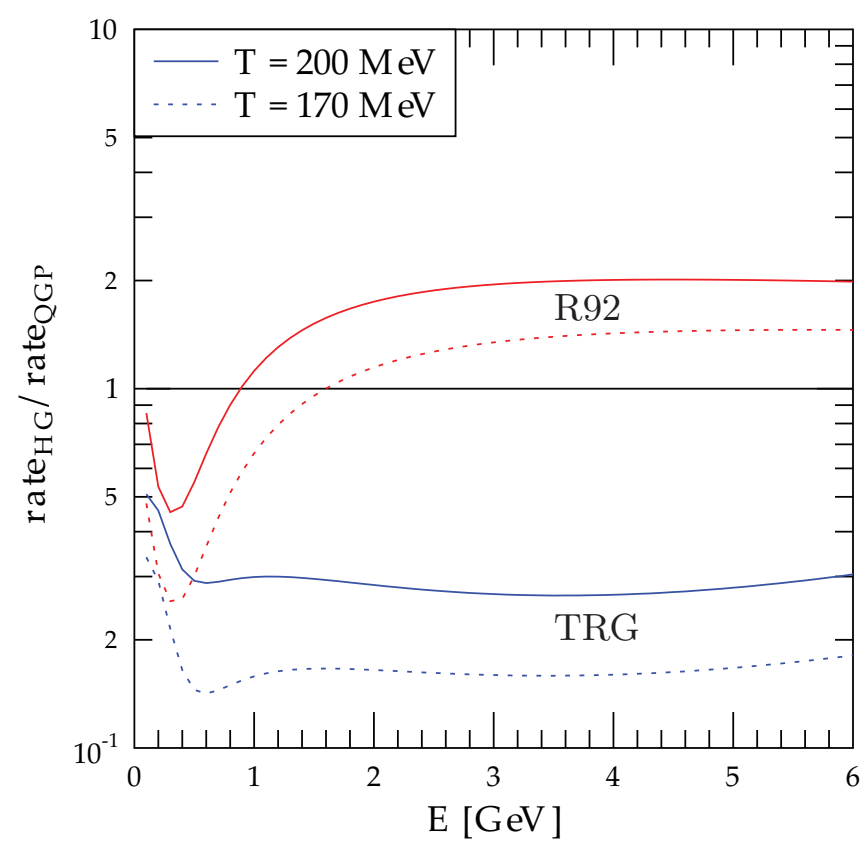

FIG. 2. (Color online) The HG-to-QGP ratio of the photon emission rates as a function of the photon energy at two different temperatures. Two different HG rates, R92 [39-41] and TRG [43], are compared.

temperatures. We see that (since both HG rates have been divided by the same QGP rate) the difference between R92 and TRG is about a factor of 6 at large energies. Furthermore, the TRG rates are always well below the QGP rates, whereas this is not the case for the $\mathrm{R} 92$ rates.

Elliptic flow for the thermal photons is calculated as in Eq. (6). Since the thermal photons cannot be distinguished from other direct photons, the elliptic flow from thermal photons alone cannot be measured. In what follows, we assume that the net contribution to the photonic $v_{2}$ from the other direct photon sources remains small, especially since the fragmentation photons with a positive $v_{2}$ should partially cancel the negative $v_{2}$ of the photons arising from parton-medium interactions [9].

If other components are emitted isotropically we can roughly estimate how much they "wash away" the elliptic flow coming from thermal photons. The total elliptic flow is then

$$
\begin{aligned}
v_{2} & =\left(\int d \phi \cos (2 \phi) \frac{d N^{\mathrm{th}}}{d p_{T}^{2} d \phi d y}\right)\left(\int d \phi \frac{d N^{\mathrm{all}}}{d p_{T}^{2} d \phi d y}\right)^{-1} \\
& =v_{2}^{\mathrm{th}}\left(\frac{d N^{\mathrm{th}}}{d p_{T}^{2} d y}\right)\left(\frac{d N^{\mathrm{all}}}{d p_{T}^{2} d y}\right)^{-1},
\end{aligned}
$$

where $d N^{\text {all }} / d p_{T}^{2} d y$ corresponds to the measured $p_{T}$ spectrum of direct photons and $v_{2}^{\text {th }}$ is the $v_{2}$ of thermal photons alone.

\section{RESULTS FOR RHIC}

\section{A. Hadron spectra and elliptic flow}

First we show the hadronic observables to demonstrate that our hydrodynamical description of the bulk QCD medium is

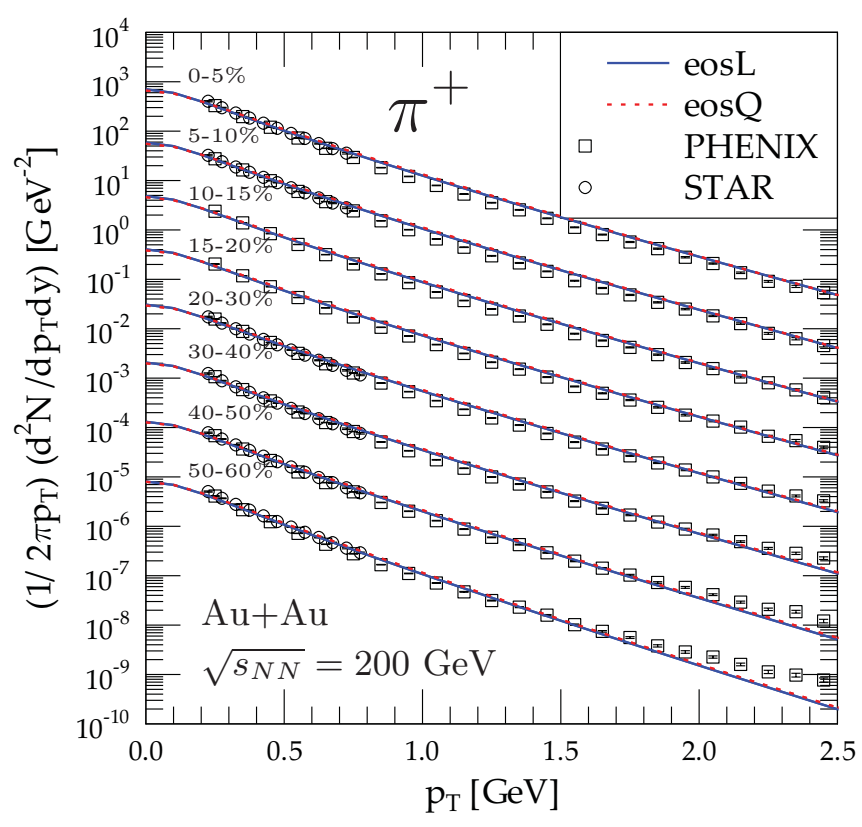

FIG. 3. (Color online) The $p_{T}$ spectra of positive pions for $\sqrt{s_{N N}}=200 \mathrm{GeV} \mathrm{Au}+\mathrm{Au}$ collisions at RHIC compared with the PHENIX data [44]. The centrality classes are indicated in the figure and the spectra are scaled by increasing powers of $10^{-1}$.

reasonable. Figure 3 shows the transverse momentum spectra of positively charged pions in different centrality classes in $\sqrt{s_{N N}}=200 \mathrm{GeV} \mathrm{Au}+\mathrm{Au}$ collisions at RHIC. As we can see, we have a good fit to the pion spectra below $p_{T} \approx 2 \mathrm{GeV}$ for a very wide range of centralities. The integrated elliptic flow of charged hadrons from Eq. (7) is plotted in Fig. 4 together with the data obtained by the STAR Collaboration [45] using the four-particle cumulant and Lee-Yang zeros (LYZ) methods, which should best reflect the elliptic flow relative to the reaction plane defined by the impact parameter. We have a fairly good description of the data also here, although the centrality dependence of the computed $v_{2}$ is not fully reproduced. We expect, however, that fine-tuning the initial density profile, invoking the Monte Carlo Glauber model and possibly also event-by-event hydrodynamics (see, e.g., Ref. [46]) as well as including viscous effects (see, e.g., Ref. [47]), will improve the agreement. These improvements are, however, beyond the scope of this exploratory paper.

\section{B. Photon spectra}

To study how much elliptic flow is washed away by other sources of direct photons at RHIC, we need to estimate the other components. We do this by fitting the measured photon $p_{T}$ spectrum. To study the uncertainties due to the chosen fit functions, we use two different forms. Our first choice (fit 1) is an exponential combined with a power-law function [20]:

$$
f\left(p_{T}\right)=A \exp \left(-p_{T} / T\right)+\frac{C}{\left(1+p_{T}^{2} / b\right)^{n}},
$$

where $A, T, C, b$, and $n$ are the fit parameters. This fit function is physically motivated by the QCD-like power-law behavior 


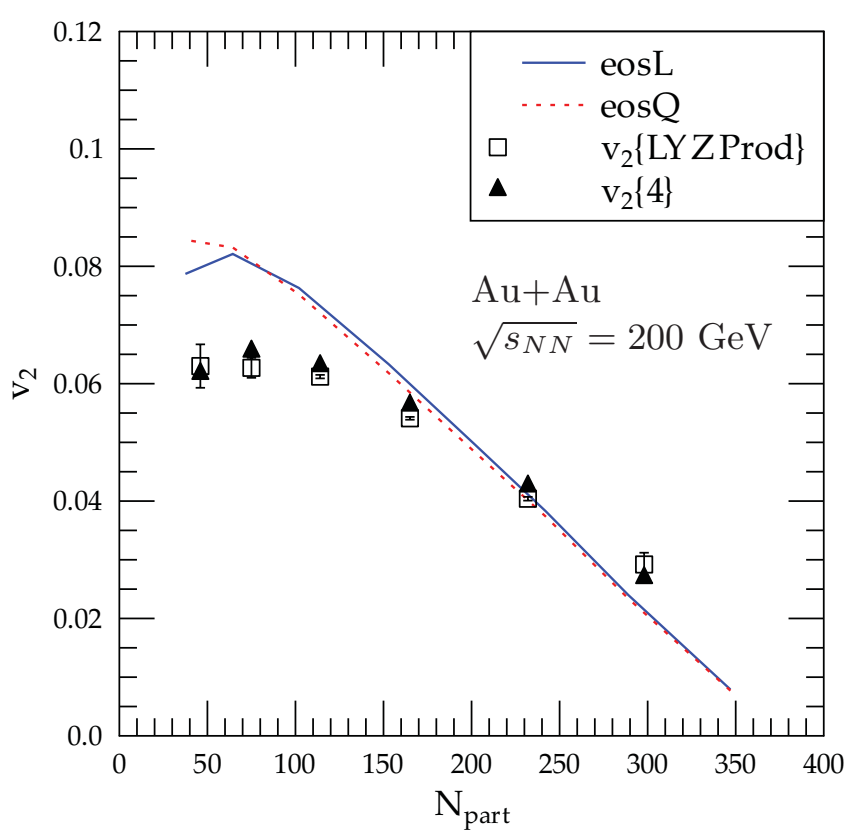

FIG. 4. (Color online) The integrated $v_{2}$ for charged hadrons in $\sqrt{s_{N N}}=200 \mathrm{GeV} \mathrm{Au}+\mathrm{Au}$ collisions at RHIC compared with the STAR data [45].

at high $p_{T}$ and the thermal-like exponential at low $p_{T}$. Our alternative choice (fit 2 ) is a mere power-law function:

$$
f\left(p_{T}\right)=\frac{C}{\left(1+p_{T}^{2} / b\right)^{n}} .
$$

In these fits, we use the photon data from the PHENIX Collaboration. The older data sets $[18,19]$ have large error bars at low $p_{T}$, but in the more recent low- $p_{T}$ data [20] the error bars are much smaller. Unfortunately, the centrality classes in these measurements differ from each other. For our fits shown in Fig. 5, we have simply combined the 0-20\% (20-40\%) centrality data from Ref. [20] with the 10-20\% (30-40\%) centrality data from Ref. [19]. In our fits we included all data points from the aforementioned sets.

With fit 1 , we first find the parameters $b$ and $n$ by fitting the measured photon $p_{T}$ spectra in $p+p$ collisions using the PHENIX data [18,20] shown in Fig. 5(a). Then for the $\mathrm{Au}+\mathrm{Au}$ case, keeping the high- $p_{T}$ slope parameters $b$ and $n$ fixed, we find $A, T$, and $C$ by fitting the PHENIX data $[19,20]$ for the two centrality classes shown in Fig. 5. For fit 2, we use the same data sets. The best fit parameters obtained for the power-law fits are listed in Table II and the parameters for fit 1 can be found in Table III. Fits 1 and 2 have equally small $\chi^{2}$ values at both centralities.

TABLE II. The parameters obtained for the power-law fits 2 .

\begin{tabular}{lccc}
\hline \hline & $C\left(\mathrm{GeV}^{-2}\right)$ & $b\left(\mathrm{GeV}^{2}\right)$ & $n$ \\
\hline$p+p$ & $3.29 \times 10^{-1}$ & $4.37 \times 10^{-1}$ & 3.09 \\
$\mathrm{Au}+\mathrm{Au} \mathrm{0-20 \%}$ & $2.16 \times 10^{14}$ & $5.24 \times 10^{-5}$ & 3.35 \\
$\mathrm{Au}+\mathrm{Au} \mathrm{20-40 \%}$ & $9.33 \times 10^{17}$ & $6.35 \times 10^{-6}$ & 3.52 \\
\hline \hline
\end{tabular}

TABLE III. The parameters for the exponential + power-law fits 1. In this case, $n$ and $b$ are obtained from Table II.

\begin{tabular}{lccc}
\hline \hline & $A\left(\mathrm{GeV}^{-2}\right)$ & $T(\mathrm{GeV})$ & $C$ \\
\hline $\mathrm{Au}+\mathrm{Au} \mathrm{0-20 \%}$ & 85.4 & 0.212 & 4.96 \\
$\mathrm{Au}+\mathrm{Au} \mathrm{20-40 \%}$ & 30.7 & 0.218 & 1.18 \\
\hline \hline
\end{tabular}

In Fig. 5(b) we replot the low- and mid- $p_{T}$ region from Fig. 5(a) and show our thermal photon results obtained with eosL (eosQ) using the TRG (R92) rates in the HG phase. For clarity, we plot the eosL results only for $T_{s}=170 \mathrm{MeV}$. If we do the switch of the emission rate at $T_{s}=200 \mathrm{MeV}$, we get $30 \%(10 \%)$ fewer photons at $p_{T}=1(2) \mathrm{GeV}$, because the TRG emission rate is smaller than the QGP emission rate, as shown in Fig. 2.

Our thermal photon results shown in Fig. 5(b) differ by a factor of 2 at high $p_{T}$. Some of this difference comes from the small difference in the initial temperature profiles, which in our case are obtained from the fixed initial entropy density through the EoS. However, a more dominant effect is the different mapping of the energy density to the temperature in eosQ and eosL. The difference in the actual temperature in the two cases is not large but the exponential temperature dependence in the emission rates magnifies the effect considerably.

From the previous photon studies (see, e.g., Refs. [11,12]), we know that photons from $\mathrm{HG}$ are contributing mostly at small $p_{T}$. In Fig. 5 the difference between the eosQ+R92 and eosL+TRG results shrinks down at low $p_{T}$ because the R92 HG emission rate is larger than that in the TRG rates and because with eosQ the HG volume becomes larger than with eosL. When we use the TRG rates and eosL, only $3 \%$ of the photons come from the $\mathrm{HG}$ at $p_{T}=1 \mathrm{GeV}$. With eosQ and the R92 rates, about $50 \%$ of the photons originate from $\mathrm{HG}$ at the same $p_{T}$.

We also note that in the low- $p_{T}$ region our thermal photon results very clearly undershoot the latest PHENIX data. We checked that changing the freeze-out temperature to $T_{\mathrm{dec}}=$ $120 \mathrm{MeV}$ gives only a negligible improvement. This feature is typical to almost all hydrodynamical calculations, as can been seen, for example, from Fig. 43 in Ref. [48].

At $p_{T} \sim 3 \mathrm{GeV}$ the obtained thermal photon emission is almost enough to match fit 1 at both centralities if eosL is considered. This suggests that we may have a window for thermal photon dominance at this $p_{T}$. However, if we compare with fit 2 , there is always at least a factor-of-2 difference. Event-by-event fluctuations in the initial state, however, were shown to increase the thermal emission at $p_{T}>2 \mathrm{GeV}$ [49], and thus we should indeed have a better chance to have a region where the direct photon $p_{T}$ spectrum, and consequently also the photon $v_{2}$, at RHIC is entirely dominated by the thermal emission.

\section{Photon elliptic flow}

In Fig. 6(a) we plot the elliptic flow of the thermal photons using both eosQ and eosL with the R92 rates. Figure 6(b) shows the same calculations but with the TRG rates. Unlike 

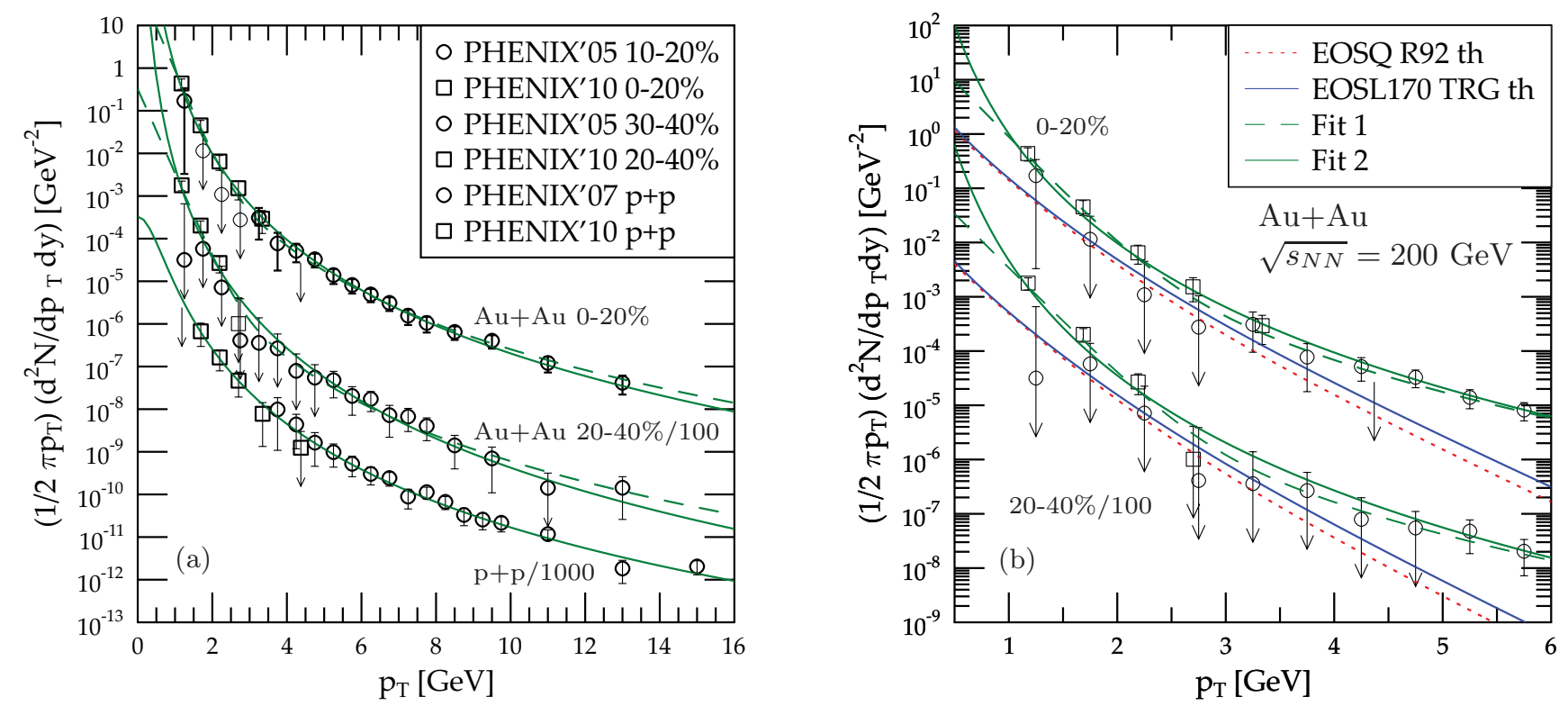

FIG. 5. (Color online) The fits 1 and 2 to the measured photon spectra for two different centralities in $\sqrt{s_{N N}}=200 \mathrm{GeV} \mathrm{Au}+\mathrm{Au}$ collisions and in $p+p$ collisions at $\sqrt{s}=200 \mathrm{GeV}$. The $20-40 \%$ centrality-class spectra are divided by 100 and the $p+p$ case by 1000 . The data are from the PHENIX Collaboration [18-20]. Our thermal photon results are shown in (b).

for hadrons, the thermal photon elliptic flow starts to decrease quickly above $p_{T} \sim 2 \mathrm{GeV}$. The reason for this is that practically all high- $p_{T}$ photons are emitted nearly isotropically in the beginning of the evolution (see, e.g., Fig. 3 in Ref. [49]), when the hydrodynamical flow effects are very small. Because the photon emission is dominated by the early times the thermal photon elliptic flow is clearly smaller than the hadronic $v_{2}\left(p_{T}\right)$, which probes the flow anisotropy only on the freeze-out surface.

From Fig. 6(a) we see that the larger switching temperature $T_{S}$ in eosL only moves the elliptic-flow peak toward higher $p_{T}$. However, as shown in Fig. 6(b), with the TRG rates there is factor-of- 2 difference in the maximum value between the eosL170 and eosL200 cases. This systematics can be deduced from Fig. 2 and hydrodynamical evolution as follows: When the system reaches the crossover region near the QCD phase transition, there has already been a significant anisotropy developed in the transverse flow which is directly reflected to the thermal photon $v_{2}$. Thus, if the photon emission is increased (decreased) in this region, the thermal photon $v_{2}$ increases (decreases). As seen in Fig. 2, with the TRG rates the QGP emission rate is larger than the $\mathrm{HG}$ rate. Hence, increasing the switching temperature decreases the emission and thus the $v_{2}$. This effect is seen in Fig. 6(b). With the R92 rates in Fig. 6(a) the situation is slightly different, as in the crossover region $T=170-200 \mathrm{MeV}$ the QGP emission rate is larger than the $\mathrm{HG}$ rate at small energies and vice versa at high energies. Thus, at low $p_{T}$ in Fig. 6(a) the situation is similar to that in Fig. 6(b) (i.e., $v_{2}$ is larger for eosL170 than for eosL200). At $p_{T}>2 \mathrm{GeV}$, the increase of the switching temperature now increases the total emission, thus making $v_{2}$ larger for eosL200 than for eosL170.

We also notice from the eosQ results in Fig. 6 that the maximum $v_{2}$ decreases by a factor of 2 when we replace the
R92 rates with the TRG rates. Because the QGP rates in both cases are the same, this signals to us that the hadron gas indeed plays an important role in generating the thermal photon $v_{2}$ in the eosQ case. To quantify this statement, we plot in Fig. 7 the fraction of photons $v_{2}\left(p_{T}\right)$ coming from the $\mathrm{HG}$ phase. We define $v_{2}^{\mathrm{HG}}$ as

$$
v_{2}^{\mathrm{HG}}=\frac{\int d \phi \cos (2 \phi) \frac{d N^{\mathrm{HG}}}{d p_{T}^{2} d \phi d y}}{\int d \phi \frac{d N^{\mathrm{QGP}+\mathrm{HG}}}{d p_{T}^{2} d \phi d y}},
$$

that is, relative to all thermal photons. We see that the photon $v_{2}$ can be mostly from the HG (eosQ with R92), mostly from the QGP (eosL170 with TRG), or between these extremes (eosQ + TRG and eosL170 + R92). Thus, both the EoS and the $\mathrm{HG}$ emission rate have a big effect on where the thermal photon $v_{2}$ originates from.

Figure 8 illustrates how much elliptic flow of thermal photons is washed away if we include other direct photon components, assuming that they are produced isotropically. We can see that the final photon $v_{2}$, obtained from Eq. (9) based on fits 1 and 2, is clearly smaller than the thermal one and also that the different fit functions modify the place and shape of the peak, keeping the maximum $v_{2}$ roughly the same, however.

In Fig. 9, we plot both the thermal photon and the full direct photon elliptic flow in $0-20 \%$ and $20-40 \%$ centrality classes for the eosQ + R92 and eosL170 + TRG cases. The latter can be considered a state-of-the-art calculation in that a realistic EoS and the latest rates are utilized. In the eosQ+R92 case, the elliptic flow is as large as it can be in our approach. For both cases, fit 1 is used to estimate how the other components reduce the elliptic flow. As seen in Fig. 5, the thermal photon yield is smaller in the eosQ case, and hence the other direct 

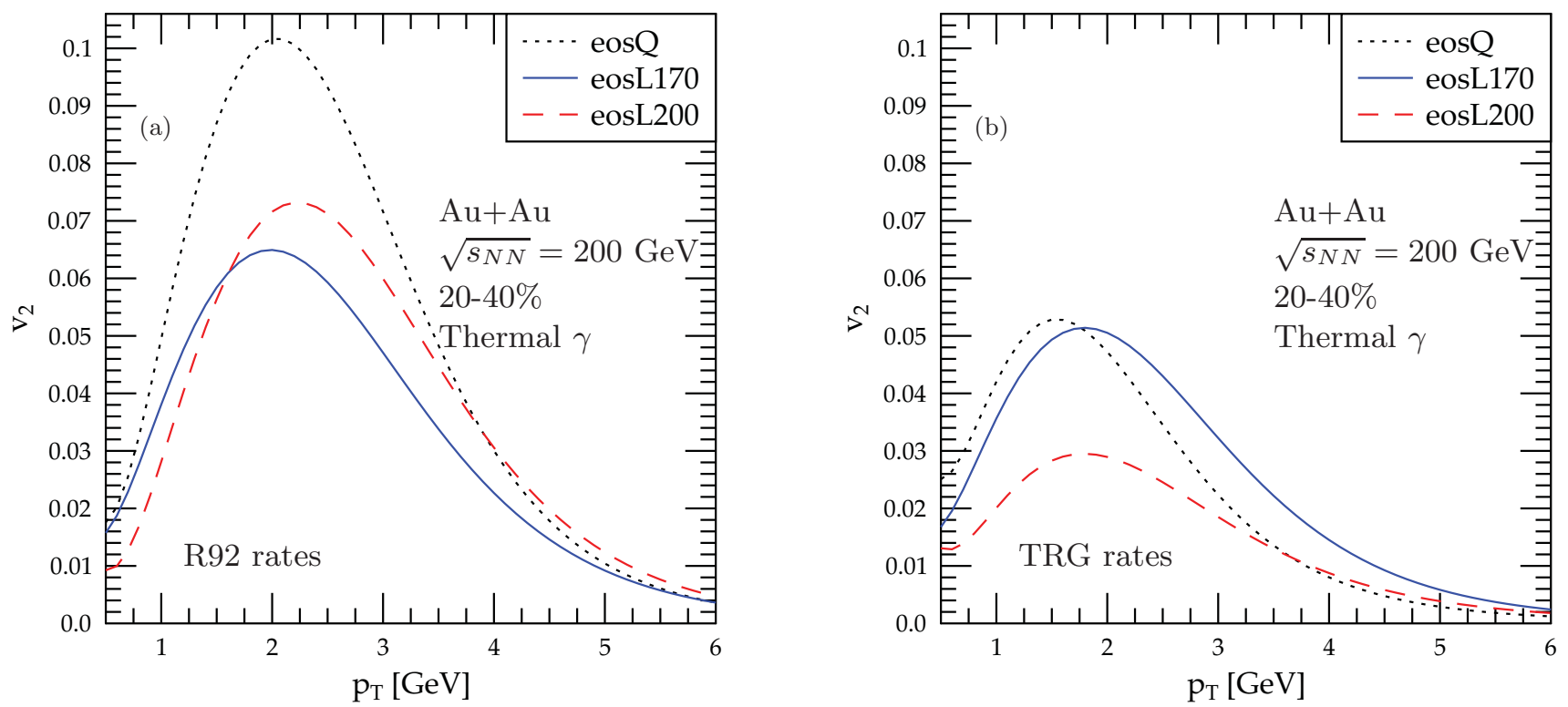

FIG. 6. (Color online) Elliptic flow of thermal photons in $\mathrm{Au}+\mathrm{Au}$ collisions at $\sqrt{s_{N N}}=200 \mathrm{GeV}$.

photon components wash away more of the elliptic flow in the eosQ case than in eosL170 case.

Finally, in Fig. 10 we compare our calculations with photon $v_{2}$ data measured by the PHENIX Collaboration [50]. ${ }^{2}$ The PHENIX result is obtained using the event plane from hadrons whereas our calculations are made with respect to the reaction plane. This means that the calculated and measured $v_{2}$ should not necessarily coincide, but if the situation is similar to

${ }^{2}$ These data were published during the review process of this paper.

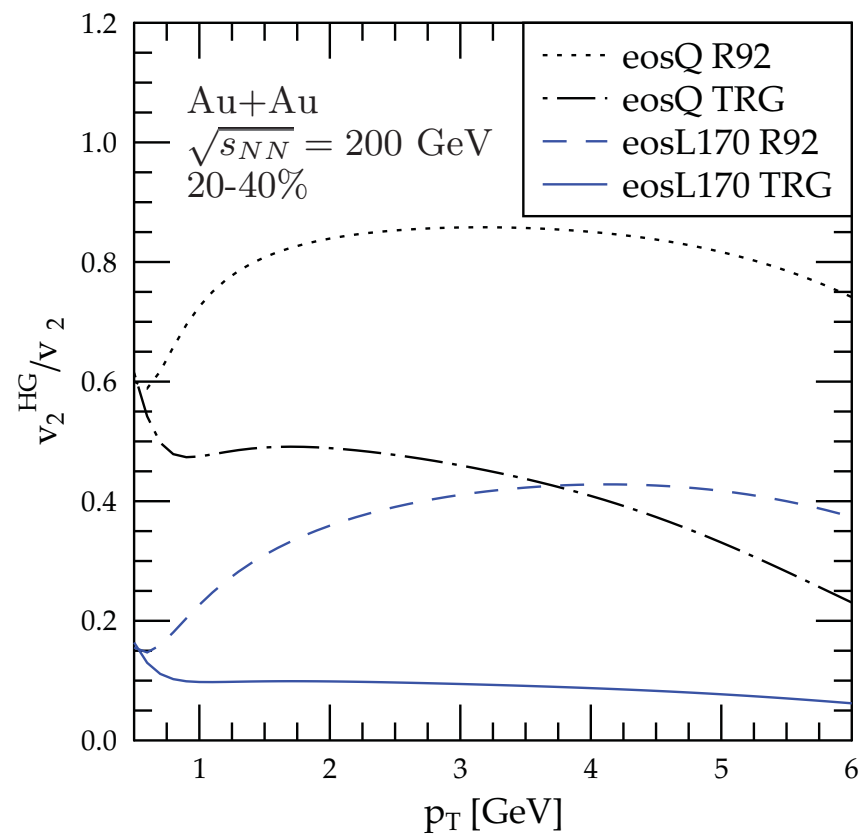

FIG. 7. (Color online) The contribution to the thermal photon elliptic flow from the hadron gas in $\mathrm{Au}+\mathrm{Au}$ collisions at $\sqrt{s_{N N}}=$ $200 \mathrm{GeV}$. the hadronic case, their difference should not be larger than $\sim 20 \%$ [46]. As seen in the figure, the measured value at the peak is much larger than the thermal photon $v_{2}$. Because even our maximal $v_{2}$ case, eosQ $+\mathrm{R} 92$, is below the data, we study how much more $v_{2}$ could be generated by lowering the decoupling temperature down to $T_{f}=120 \mathrm{MeV}$. We emphasize, however, that with this low $T_{f}$ the hydrodynamical calculation overshoots the measured pion $p_{T}$ spectra [24]. We observe that even with a lower $T_{f}$ the thermal photon $v_{2}$ remains too small. Also the eosL170 + TRG results with two different $T_{f} \mathrm{~s}$ are shown in the figure.

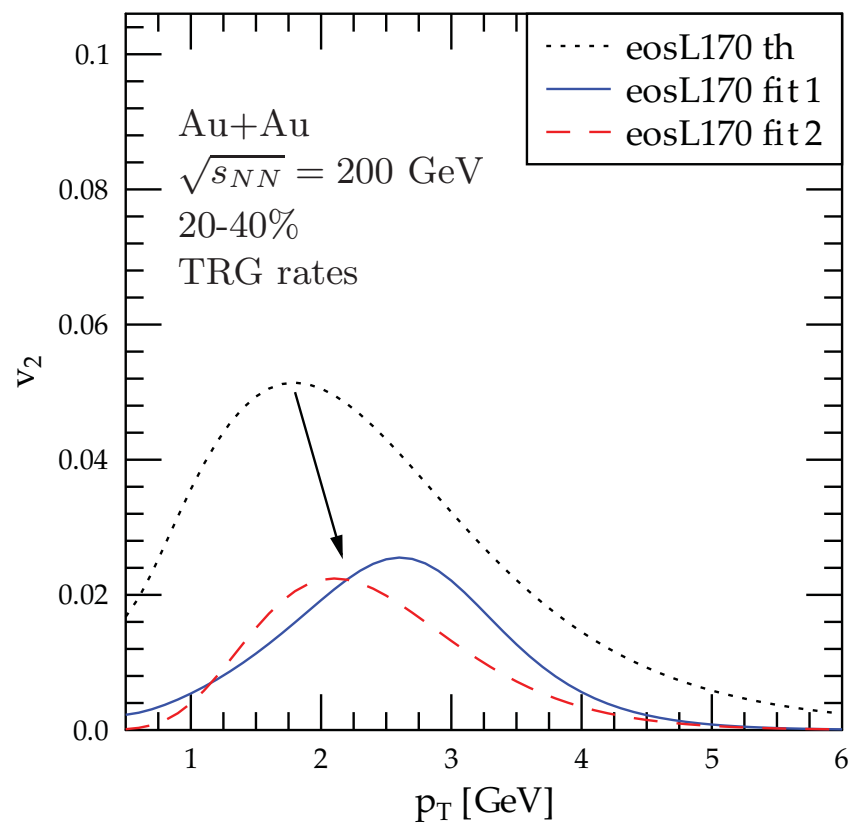

FIG. 8. (Color online) Elliptic flow of thermal and direct photons in $\mathrm{Au}+\mathrm{Au}$ collisions at $\sqrt{s_{N N}}=200 \mathrm{GeV}$. 


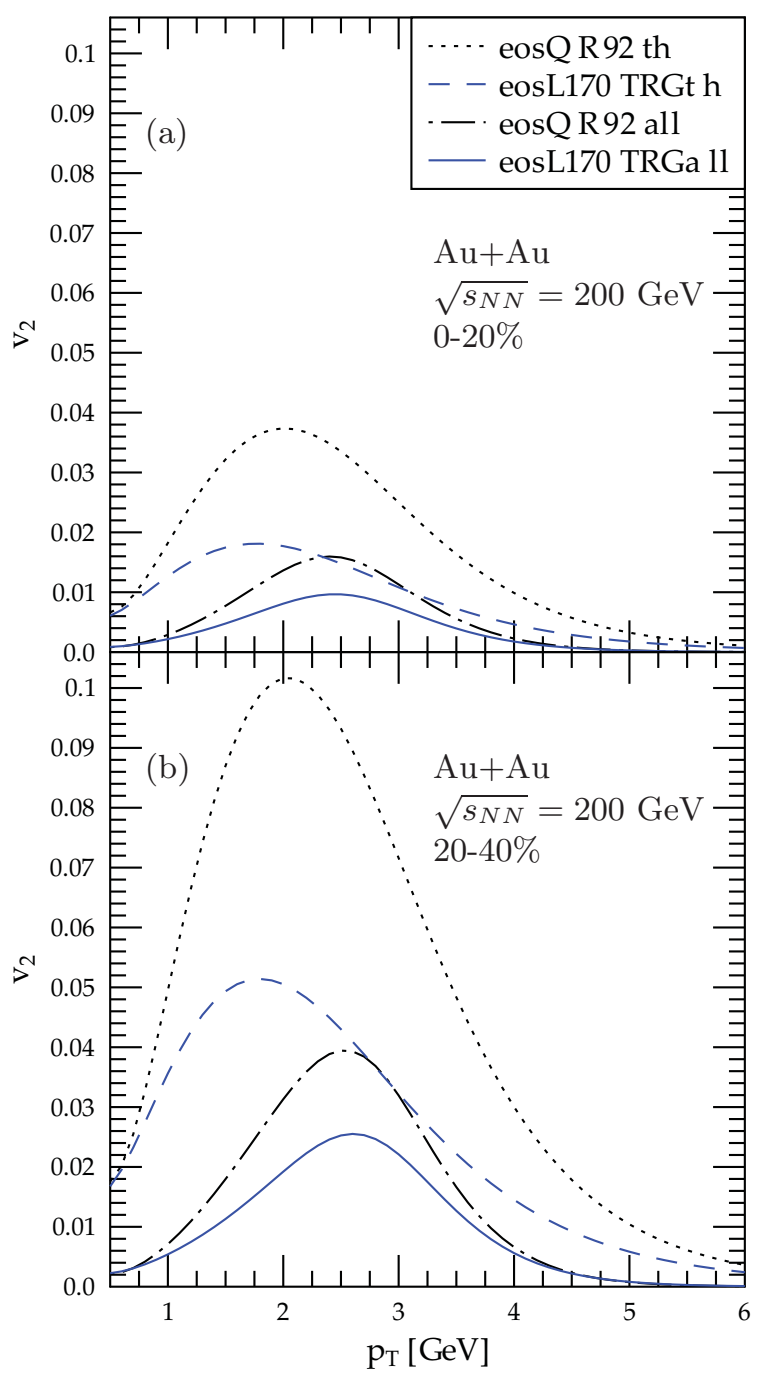

FIG. 9. (Color online) Elliptic flow of direct photons in $\mathrm{Au}+\mathrm{Au}$ collisions at $\sqrt{s_{N N}}=200 \mathrm{GeV}$.

\section{PREDICTION FOR THE LHC}

Next, we extrapolate our hydrodynamical modeling to the $\sqrt{s_{N N}}=2.76 \mathrm{TeV} \mathrm{Pb}+\mathrm{Pb}$ collisions at the LHC. We choose the same sWN initial density profile and decoupling temperatures as at RHIC, and, as explained in Sec. II B, use the measured charged-hadron multiplicity [26] to fix the initial entropy and initial time through the EKRT model (for details, see Ref. [27]). As seen in Fig. 11, a reasonable agreement with the measured charged-hadron $p_{T}$ spectrum [51] follows up to $p_{T} \sim 4 \mathrm{GeV}$. This ensures that our thermal photon calculations are meaningful also at the LHC energies.

In Fig. 12 we plot our prediction for the thermal photon $p_{T}$ spectrum in the $\sqrt{s_{N N}}=2.76 \mathrm{TeV} \mathrm{Pb}+\mathrm{Pb}$ collisions at the LHC. The bands shown are defined by the cases eosL170 + R92 and eosQ + TRG, which give the largest and smallest yields, correspondingly. We see that the uncertainty coming from the EoS and from the HG emission rates is at its largest, of the order of $40 \%$. We note that these predictions are qualitatively quite similar to the predictions given in Refs. $[21,22]$ for $\sqrt{s_{N N}}=5.5 \mathrm{TeV}$.

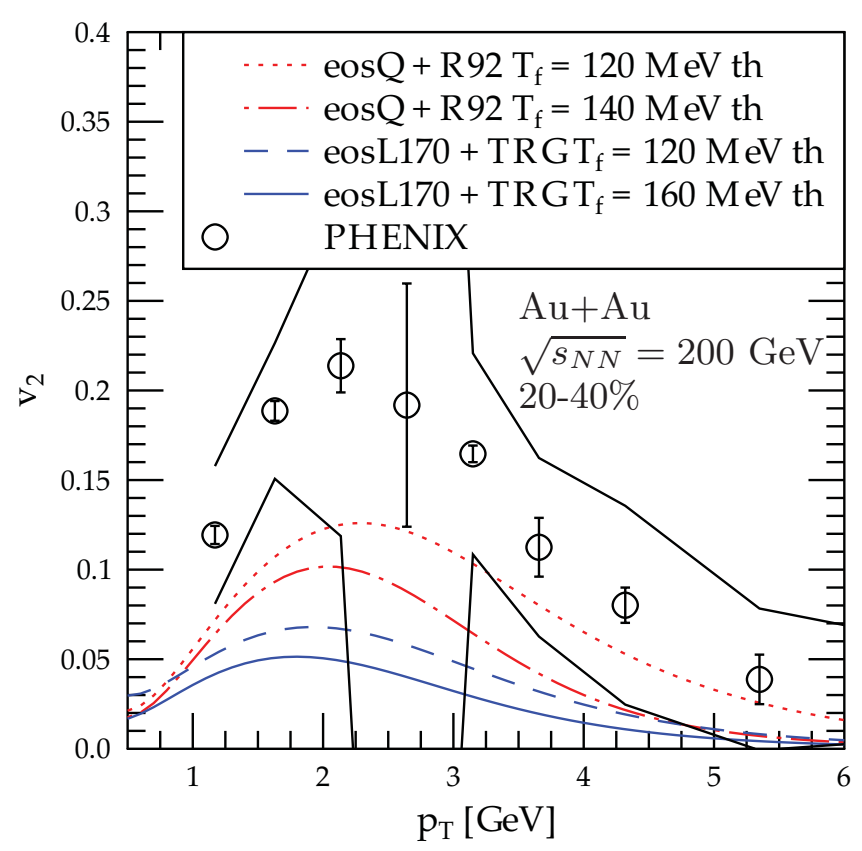

FIG. 10. (Color online) Elliptic flow of thermal photons in $\mathrm{Au}+$ Au collisions at $\sqrt{s_{N N}}=200 \mathrm{GeV}$ with two different freeze-out temperatures compared with the PHENIX Collaboration measurement [50].

Since currently we do not have the measured total direct photon spectrum at the LHC available yet (as we had at RHIC), we can consider only the thermal photon elliptic flow here. It is, however, very interesting to compare the thermal photon elliptic flow with the RHIC results. We plot the obtained thermal photon $v_{2}$ in Fig. 13 for $20-40 \%$ central collisions. We can see that, similarly to the hadronic case [52], the

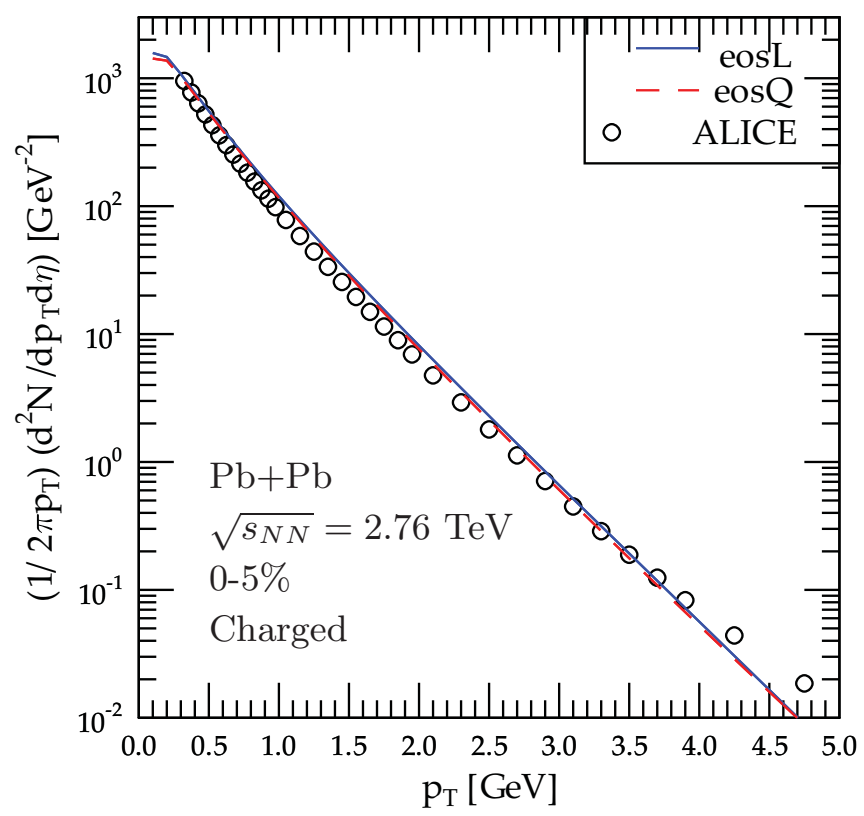

FIG. 11. (Color online) Transverse momentum spectra of charged hadrons in $0-5 \%$ most central $\mathrm{Pb}+\mathrm{Pb}$ collisions at $\sqrt{s_{N N}}=$ 2.76 TeV. Data from the ALICE Collaboration [51]. 


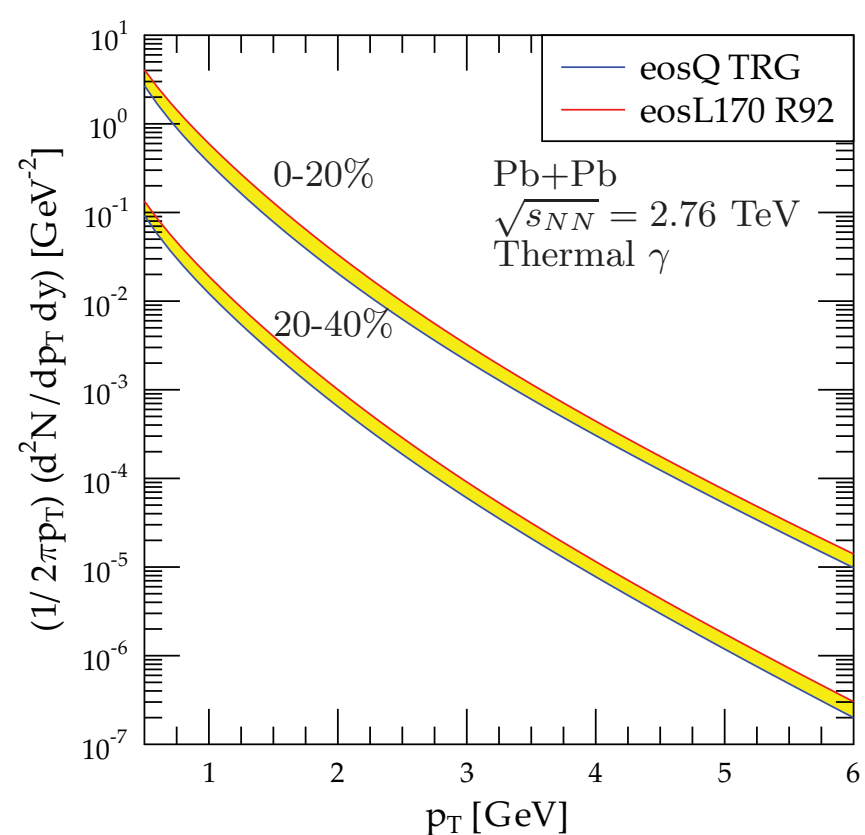

FIG. 12. (Color online) Transverse momentum spectra of thermal photons in $\mathrm{Pb}+\mathrm{Pb}$ collisions at $\sqrt{s_{N N}}=2.76 \mathrm{TeV}$.

thermal photon elliptic flow is very similar at RHIC and LHC. This is a nontrivial result because the temperature range, flow range, as well as the volume factors for photon emission (from the QGP in particular) are larger at the LHC than at RHIC, and, as discussed in Ref. [25], also the flow asymmetry near the phase transition region is larger at the LHC. Then, when going from RHIC to LHC, to arrive at a similar $v_{2}$ in both cases, the increased flow asymmetry in the numerator of Eq. (6) is compensated by the increased photon yields in the denominator of Eq. (6). In Fig. 14 we again plot the hadronic fraction of $v_{2}\left(p_{T}\right)$ for the same cases as in Fig. 7. The figure shows that $v_{2}^{\mathrm{HG}}$ is again very close to the corresponding fraction at RHIC.

\section{DISCUSSION}

We considered the sensitivity of thermal photon production to the EoS and emission rates in heavy-ion collisions at RHIC and LHC. We compared the obtained thermal photon yields with the PHENIX measurements and showed that in the window $2 \lesssim p_{T} \lesssim 3 \mathrm{GeV}$ the thermal contribution, computed with a realistic EoS (eosL) and the latest emission rates (TRG), is reasonably close to the data. As in most previous hydrodynamical studies, in the region $p_{T} \sim 1 \mathrm{GeV}$, however, we get a clearly smaller yield than what was measured most recently. We show that around $p_{T} \sim 2 \mathrm{GeV}$ the thermal photon elliptic flow peaks at a fairly large value, $5 \%$ in the $20-40 \%$ centrality class with eosL + TRG, but also that the possible other components may wash even half of this away. We emphasize, however, that the amount of $v_{2}$ washout depends on the thermal photon contribution relative to the other components. Thermal photon production near $p_{T} \sim 2 \mathrm{GeV}$ at RHIC can be expected to increase further once the

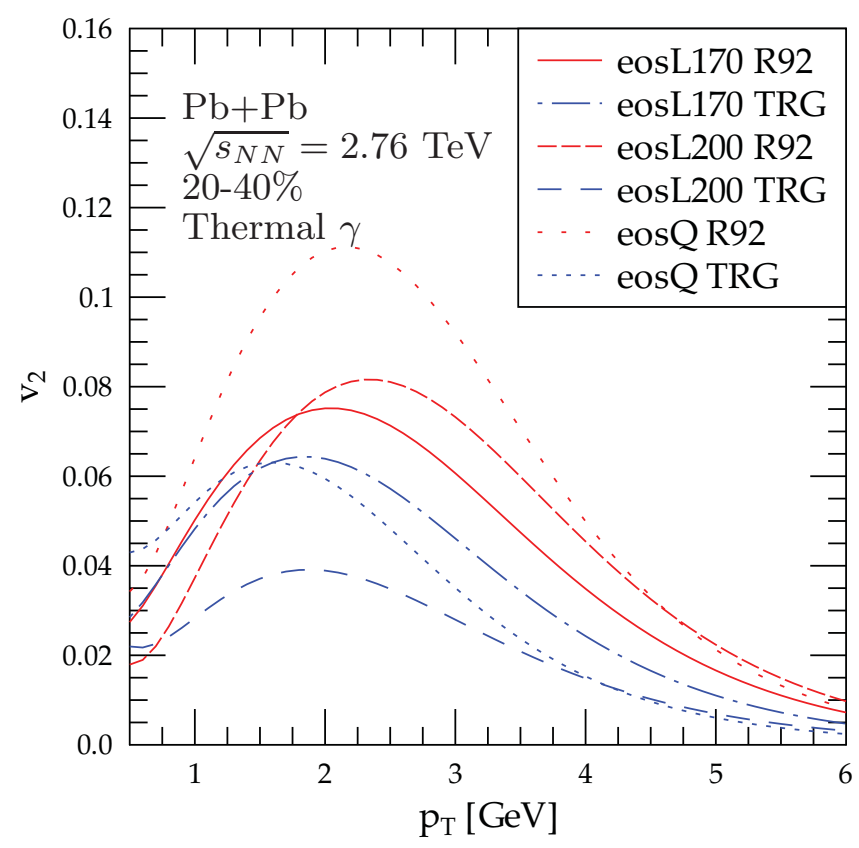

FIG. 13. (Color online) Elliptic flow of thermal photons in $\mathrm{Pb}+$ $\mathrm{Pb}$ collisions at $\sqrt{s_{N N}}=2.76 \mathrm{TeV}$.

event-by-event QCD-matter density fluctuations are accounted for [49], in which case the thermal photon production can become dominant and the $v_{2}$ washout can decrease or even vanish.

The direct photon elliptic flow measured by the PHENIX Collaboration [50] is much higher than any of our results. Excitingly, this suggests the dominance of thermal photons

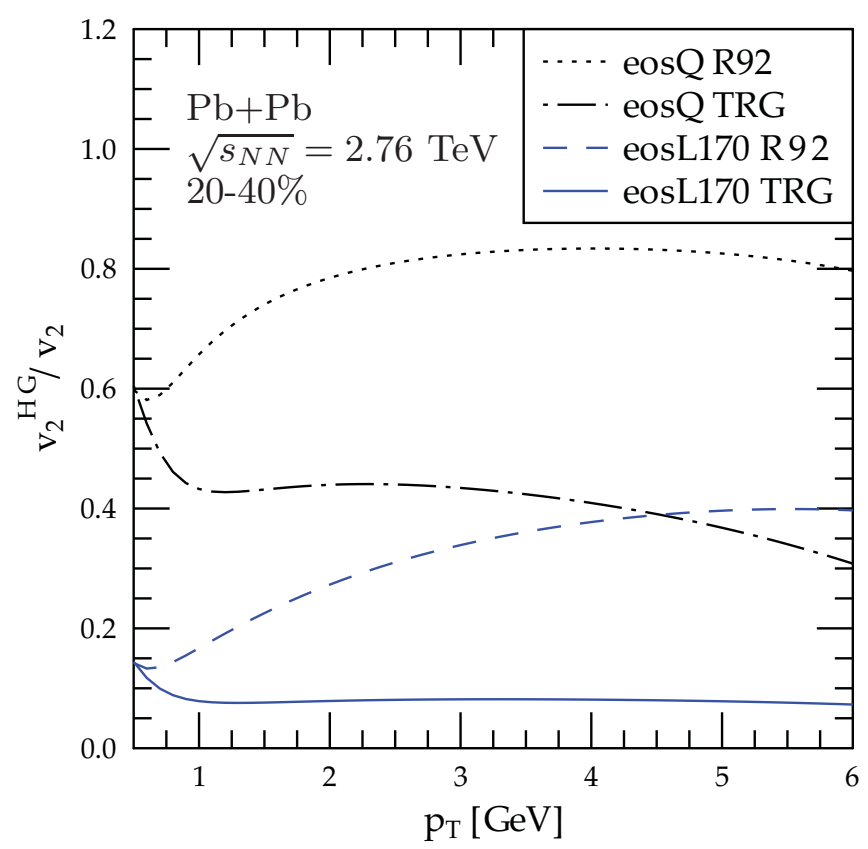

FIG. 14. (Color online) The contribution to the thermal photon elliptic flow from the hadron gas in $\mathrm{Pb}+\mathrm{Pb}$ collisions at $\sqrt{s_{N N}}=$ $2.76 \mathrm{TeV}$. 
near $p_{T} \sim 2 \mathrm{GeV}$. However, the origin of such large $v_{2}$ cannot be explained in the current framework.

Constraining our hydrodynamical modeling with the measured charged-hadron spectrum in $\sqrt{s_{N N}}=2.76 \mathrm{TeV} \mathrm{Pb}+\mathrm{Pb}$ collisions at the LHC, we predicted the thermal photon $p_{T}$ spectra and $v_{2}$. According to our results, elliptic flow of thermal photons at the LHC and RHIC are very similar in the few- $p_{T}$ region. However, one should keep in mind that we cannot fully explain the direct photon $v_{2}$ at RHIC. For the determination of a possible thermal photon window, and consequently thermal photon $v_{2}$, it will be extremely interesting to see the direct photon data at the LHC.

Next, one should consider the effects of event-by-event density fluctuations [46,53-57] on thermal photon elliptic flow. On the theoretical side, one would need a better understanding of how the degrees of freedom in the QGP would be best accounted for when computing thermal photon production, as well as a better control over the photon emission in the phase-transition region. Also the dissipative hydrodynamical effects to thermal photon production should be studied further; so far only the very first steps have been taken in this direction (see Ref. [58]).

\section{ACKNOWLEDGMENTS}

We gratefully acknowledge financial support by the Academy of Finland, KJE's Project No. 133005. In addition, H.H. is supported by the national Graduate School of Particle and Nuclear Physics. We acknowledge CSC-IT Center for Science in Espoo, Finland, for the allocation of computational resources. We would like to thank $\mathrm{R}$. Chatterjee for useful discussions.
[1] PHENIX Collaboration, S. S. Adler et al., Phys. Rev. Lett. 96, 202301 (2006).

[2] P. Aurenche, J.-P. Guillet, E. Pilon, M. Werlen, and M. Fontannaz, Phys. Rev. D 73, 094007 (2006).

[3] F. Arleo, K. J. Eskola, H. Paukkunen, and C. A. Salgado, J. High Energy Phys. 04 (2011) 055.

[4] K. J. Eskola, H. Paukkunen, and C. A. Salgado, J. High Energy Phys. 04 (2009) 065.

[5] R. J. Fries, B. Muller, and D. K. Srivastava, Phys. Rev. Lett. 90, 132301 (2003).

[6] R. J. Fries, B. Muller, and D. K. Srivastava, Phys. Rev. C 72, 041902 (2005).

[7] S. Turbide, C. Gale, E. Frodermann, and U. Heinz, Phys. Rev. C 77, 024909 (2008).

[8] F.-M. Liu, T. Hirano, K. Werner, and Y. Zhu, Phys. Rev. C 79, 014905 (2009).

[9] G.-Y. Qin, J. Ruppert, C. Gale, S. Jeon, and G. D. Moore, Phys. Rev. C 80, 054909 (2009).

[10] P. Huovinen, P. V. Ruuskanen, and J. Sollfrank, Nucl. Phys. A 650, 227 (1999).

[11] P. Huovinen, P. V. Ruuskanen, and S. S. Räsänen, Phys. Lett. B 535, 109 (2002).

[12] S. S. Räsänen, Nucl. Phys. A 715, 717 (2003).

[13] D. G. d'Enterria and D. Peressounko, Eur. Phys. J. C 46, 451 (2006).

[14] R. Chatterjee, E. S. Frodermann, U. W. Heinz, and D. K. Srivastava, Phys. Rev. Lett. 96, 202302 (2006).

[15] R. Chatterjee and D. K. Srivastava, Phys. Rev. C 79, 021901 (2009).

[16] F.-M. Liu, T. Hirano, K. Werner, and Y. Zhu, Phys. Rev. C 80, 034905 (2009).

[17] N. Armesto et al., J. Phys. G 35, 054001 (2008).

[18] PHENIX Collaboration, S. S. Adler et al., Phys. Rev. Lett. 98, 012002 (2007).

[19] PHENIX Collaboration, S. S. Adler et al., Phys. Rev. Lett. 94, 232301 (2005).

[20] PHENIX Collaboration, A. Adare et al., Phys. Rev. Lett. 104, 132301 (2010).

[21] F. Arleo et al., arXiv:hep-ph/0311131.

[22] K. Dusling and I. Zahed, Phys. Rev. C 82, 054909 (2010).

[23] K. J. Eskola, K. Kajantie, P. V. Ruuskanen, and K. Tuominen, Nucl. Phys. B 570, 379 (2000).
[24] K. J. Eskola, H. Honkanen, H. Niemi, P. V. Ruuskanen, and S. S. Rasanen, Phys. Rev. C 72, 044904 (2005).

[25] H. Niemi, K. J. Eskola, and P. V. Ruuskanen, Phys. Rev. C 79, 024903 (2009).

[26] ALICE Collaboration, K. Aamodt et al., Phys. Rev. Lett. 105, 252301 (2010).

[27] T. Renk, H. Holopainen, R. Paatelainen, and K. J. Eskola, Phys. Rev. C 84, 014906 (2011).

[28] STAR Collaboration, B. I. Abelev et al., Phys. Rev. C 79, 034909 (2009).

[29] P. F. Kolb, U. W. Heinz, P. Huovinen, K. J. Eskola, and K. Tuominen, Nucl. Phys. A 696, 197 (2001).

[30] J. P. Boris and D. L. Book, J. Comput. Phys. A11, 38 (1973).

[31] S. T. Zalesak, J. Comput. Phys. A 31, 335 (1979).

[32] J. Sollfrank, P. Huovinen, M. Kataja, P. V. Ruuskanen, M. Prakash, and R. Venugopalan, Phys. Rev. C 55, 392 (1997).

[33] M. Laine and Y. Schroder, Phys. Rev. D 73, 085009 (2006).

[34] P. Huovinen and P. Petreczky, Nucl. Phys. A 837, 26 (2010).

[35] F. Cooper and G. Frye, Phys. Rev. D 10, 186 (1974).

[36] P. B. Arnold, G. D. Moore, and L. G. Yaffe, J. High Energy Phys. 11 (2001) 057.

[37] P. B. Arnold, G. D. Moore, and L. G. Yaffe, J. High Energy Phys. 12 (2001) 009.

[38] F. Karsch, Z. Phys. C 38, 147 (1988).

[39] J. I. Kapusta, P. Lichard, and D. Seibert, Phys. Rev. D 44, 2774 (1991).

[40] L. Xiong, E. V. Shuryak, and G. E. Brown, Phys. Rev. D 46, 3798 (1992).

[41] H. Nadeau, J. I. Kapusta, and P. Lichard, Phys. Rev. C 45, 3034 (1992).

[42] C. Song, Phys. Rev. C 47, 2861 (1993).

[43] S. Turbide, R. Rapp, and C. Gale, Phys. Rev. C 69, 014903 (2004).

[44] PHENIX Collaboration, S. S. Adler et al., Phys. Rev. C 69, 034909 (2004).

[45] STAR Collaboration, B. I. Abelev et al., Phys. Rev. C 77, 054901 (2008).

[46] H. Holopainen, H. Niemi, and K. J. Eskola, Phys. Rev. C 83, 034901 (2011).

[47] P. Romatschke and U. Romatschke, Phys. Rev. Lett. 99, 172301 (2007). 
[48] PHENIX Collaboration, A. Adare et al., Phys. Rev. C 81, 034911 (2010).

[49] R. Chatterjee, H. Holopainen, T. Renk, and K. J. Eskola, Phys. Rev. C 83, 054908 (2011).

[50] PHENIX Collaboration, A. Adare et al., arXiv:1105.4126, Phys. Rev. Lett. (to be published).

[51] ALICE Collaboration, K. Aamodt et al., Phys. Lett. B 696, 30 (2011).

[52] ALICE Collaboration, K. Aamodt et al., Phys. Rev. Lett. 105, 252302 (2010).
[53] R. P. G. Andrade, F. Grassi, Y. Hama, T. Kodama, and W. L. Qian, Phys. Rev. Lett. 101, 112301 (2008).

[54] H. Petersen and M. Bleicher, Phys. Rev. C 81, 044906 (2010).

[55] K. Werner, I. Karpenko, T. Pierog, M. Bleicher, and K. Mikhailov, Phys. Rev. C 82, 044904 (2010).

[56] B. Schenke, S. Jeon, and C. Gale, Phys. Rev. Lett. 106, 042301 (2011).

[57] Z. Qiu and U. W. Heinz, Phys. Rev. C 84, 024911 (2011).

[58] K. Dusling, Nucl. Phys. A 839, 70 (2010). 